\title{
Robert Goldblatt Maps and Monads for Modal Frames
}

\author{
Dedicated to the memory of Willem Johannes Blok.
}

\begin{abstract}
The category-theoretic nature of general frames for modal logic is explored. A new notion of "modal map" between frames is defined, generalizing the usual notion of bounded morphism/p-morphism. The category Fm of all frames and modal maps has reflective subcategories $\mathbf{C H F m}$ of compact Hausdorff frames, DFm of descriptive frames, and UEFm of ultrafilter enlargements of frames. All three subcategories are equivalent, and are dual to the category of modal algebras and their homomorphisms.

An important example of a modal map that is typically not a bounded morphism is the natural insertion of a frame $A$ into its ultrafilter enlargement $E A$. This map is used to show that $E A$ is the free compact Hausdorff frame generated by $A$ relative to $\mathbf{F m}$. The monad $\mathbb{E}$ of the resulting adjunction is examined and its Eilenberg-Moore category is shown to be isomorphic to CHFm. A categorical equivalence between the Kleisli category of $\mathbb{E}$ and UEFm is defined from a construction that assigns to each frame $A$ a frame $A^{*}$ that is "image-closed" in the sense that every point-image $\{b: a R b\}$ in $A$ is topologically closed. $A^{*}$ is the unique image-closed frame having the same ultrafilter enlargement as $A$.

These ideas are connected to a category $\mathfrak{W}$ shown by S. K. Thomason to be dual to the category of complete and atomic modal algebras and their homomorphisms. $\mathfrak{W}$ is the full subcategory of the Kleisli category of $\mathbb{E}$ based on the Kripke frames.
\end{abstract}

Keywords: modal map, bounded morphism, descriptive frame, equivalence of categories, duality, reflective subcategory, monad, Kleisli category

\section{Introduction and Summary}

This paper seeks to clarify the category-theoretic nature of the relational structures (frames) that are used in the semantics of propositional modal logics. Previous work in this direction includes the author's demonstration [5] that the category MA of modal algebras and homomorphisms is dually equivalent to the category DFm whose objects are the descriptive frames and whose arrows are bounded morphisms (also called p-morphisms), these being the class of modal-validity preserving functions that have become the standard notion of "arrow" for frames. At about the same time, Thomason [9] studied the subcategory of MA whose objects are the complete and

July 22,2004

Studia Logica 0: 1-25, 0.

(C) 0 Kluwer Academic Publishers. Printed in the Netherlands. 
atomic modal algebras, and showed that it is dual to a category $\mathfrak{W}$ whose objects are the Kripke frames and whose arrows are certain ultrafilter-valued functions on frames. These unusual $\mathfrak{W}$-arrows do not appear to have been the subject of further attention.

The focus of our work is the notion of the ultrafilter enlargement EA of a frame $A$. Each frame has a dual algebra based on its collection of internal subsets, while conversely each modal algebra has a dual frame based on its collection of ultrafilters. Composing these constructions produces the frame $E A$ as the "double-dual" of $A$. Now the internal subsets of any frame form a base for a topology, and in its topology $E A$ is compact, Hausdorff and image-closed, the latter meaning that each point-image, i.e. each subset of $A$ of the form $\{b: a R b\}$, is topologically closed. These three properties together define the descriptive frames.

Another important operation assigns to each frame $A$ an image-closed frame $A^{*}$ having the same underlying set and internal subsets as $A$, and the same ultrafilter enlargement, i.e. $E\left(A^{*}\right)=E A$. We will see that image-closed frames with the same ultrafilter enlargement are identical. This implies that if $E A=E B$ then $A^{*}=B^{*}$, for any frames $A, B$. In general $A^{*}$ is the only image-closed frame having the same ultrafilter enlargement as $A$.

One of our main aims is to explain how $E A$ is freely generated by $A$, in the same sense in which each set freely generates an algebra of any given equational type. We also wish to clarify the nature of Thomason's category $\mathfrak{W}$ and place it into context. An immediate obstacle that must be addressed here is that, although there is a natural insertion $\eta_{A}: A \rightarrow E A$ of each frame into its ultrafilter enlargement, these insertion functions are typically not bounded morphisms. In fact we will show that $\eta_{A}$ is a bounded morphism iff each point-image is a compact subset of $A$. This compels us to work with a new notion of modal map $f: A \rightarrow B$ between frames, a notion that is weaker than that of bounded morphism, but still strong enough to ensure that $f$ induces a homomorphism from the dual algebra of $B$ to the dual algebra of $A$. The frames and their modal maps form a category $\mathbf{F m}$ in which each $\eta_{A}$ is present as an arrow from $A$ to $E A$. Moreover, the identity function on $A$ is always an isomorphism in $\mathbf{F m}$ from $A$ to $A^{*}$, but is not a bounded morphism from $A$ to $A^{*}$ unless $A$ is image-closed, in which case $A=A^{*}$. If $A$ is compact and Hausdorff, then $\eta_{A}$ is an isomorphism from $A$ onto $E A$, but is only a bounded morphism when $A$ is descriptive.

$E A$ can now be characterized as the free compact Hausdorff frame generated by $A$, in the sense that any modal map $f$ from $A$ into a compact Hausdorff frame $B$ has a unique extension along $\eta_{A}$ to a modal map $\tilde{f}$ from 
$E A$ to $B$, i.e. $f=\tilde{f} \circ \eta_{A}$ :

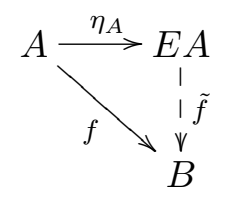

$\mathbf{F m}$ has three subcategories of interest to us. These are the categories CHFm of compact Hausdorff frames, DFm of descriptive frames, and UEFm of ultrafilter enlargements of frames. The arrows in all three categories are the modal maps between their relevant objects, but it turns out that all arrows in $\mathbf{D F m}$ and its subcategory $\mathbf{U E F m}$ are bounded morphisms: any modal map between descriptive frames is bounded. Although the three subcategories are distinct, they are all equivalent in the category-theoretic sense. Equivalence means that between any two of these categories there are functors in both directions that compose to give natural isomorphisms with the identity functors on the categories. This follows because each CHFmobject $A$ is isomorphic to the $\mathbf{U E F m}$-object $E A$, a fact that depends on the availability of modal maps and not just bounded morphisms.

The $E A$-construction provides a left adjoint to the inclusion functor from CHFm to $\mathbf{F m}$, showing that $\mathbf{C H F m}$ is a reflective subcategory of $\mathbf{F m}$. Associated with this adjunction is a categorical structure $\mathbb{E}$ known as a monad. This in turn gives rise to two further categories connected with $\mathbf{F m}$, the Eilenberg-Moore category $\mathbf{F m}^{\mathbb{E}}$ and the Kleisli category $\mathbf{F m}_{\mathbb{E}}$. These have comparison functors $K: \mathbf{C H F m} \rightarrow \mathbf{F m}{ }^{\mathbb{E}}$ and $L: \mathbf{F m}_{\mathbb{E}} \rightarrow \mathbf{C H F m}$.

Now the theory of monads tells us that whenever a monad arises from a reflective subcategory, then its Eilenberg-Moore and Kleisli categories are equivalent to each other and to the reflective subcategory. Thus $\mathbf{F m}{ }^{\mathbb{E}}$ and $\mathbf{F} \mathbf{m}_{\mathbb{E}}$ are equivalent to $\mathbf{C H F m}$ on general grounds. It follows that all five categories

\section{CHFm, DFm, UEFm, Fm $_{\mathbb{E}}, \mathbf{F m}^{\mathbb{E}}$}

are equivalent, and all are dual to the category MA of modal algebras.

But in this case of modal frames two stronger observations can be made. First, $\mathbf{C H F m}$ is not just equivalent to $\mathbf{F m}^{\mathbb{E}}$ : the two categories are isomorphic, and the isomorphism is given by the comparison functor $K$. The objects of $\mathbf{F m}^{\mathbb{E}}$ are the so-called $\mathbb{E}$-algebras $(A, \xi)$, comprising an $\mathbf{F m}$-object $A$ and an $\mathbf{F m}$-arrow $\xi: E A \rightarrow A$ satisfying certain conditions. In our frame case the functor $K$ has $K A=\left(A, \varepsilon_{A}\right)$, where for compact Hausdorff $A$ the modal map $\varepsilon_{A}: E A \rightarrow A$ is the inverse of the isomorphism $\eta_{A}: A \rightarrow E A$. 
We will show that the $\mathbb{E}$-algebras are precisely the pairs $\left(A, \varepsilon_{A}\right)$ with $A$ compact Hausdorff, and that $K$ acts bijectively between the objects and arrows of CHFm and $\mathbf{F m}{ }^{\mathbb{E}}$.

The general theory also tells us that the comparison functor $L$ makes the Kleisli category $\mathbf{F m}_{\mathbb{E}}$ equivalent to the image of the left adjoint $E: \mathbf{F m} \rightarrow$ CHFm. In our case this image is the category UEFm of all ultrafilter enlargements of frames. Demonstrating the equivalence in general may require the axiom of choice. The category $\mathbf{F m}_{\mathbb{E}}$ has the same objects as $\mathbf{F m}$, with an $\mathbf{F m}_{\mathbb{E}}$ arrow from $A$ to $B$ being an $\mathbf{F m}$-arrow from $A$ to $E B$. The functor $L$ takes an $\mathbf{F m}_{\mathbb{E}}$-object $A$ to $E A$. $L$ acts bijectively on arrows, but it may not be bijective on objects. We can see why in our frame case from the fact that if a frame $A$ is not image-closed, then we have $E A=E\left(A^{*}\right)$ but $A \neq A^{*}$. But given an object $C$ in the image of $E$, we can choose some $A$ such that $C=E A=L A$ and put $J C=A$. The bijective action of $L$ on arrows then inverts to extend this to a functor $J: \mathbf{U E F m} \rightarrow \mathbf{F m}_{\mathbb{E}}$ establishing an equivalence with $L$.

However, and this is our second observation, in the case of modal frames there is no need to use choice here. Instead we can make use of the "imageclosure" construction $A^{*}$. Since $E A=E B$ implies $A^{*}=B^{*}$ in general, putting $J(E A)=A^{*}$ gives a well-defined function on $\mathbf{U E F m}$-objects which extends to a suitable functor $J$.

The details of all these claims are set out below, where the desired explanation of the nature of Thomason's cateogry $\mathfrak{W}$ will also be provided. It will be shown that $\mathfrak{W}$ is precisely the subcategory of the Kleisli category $\mathbf{F m}_{\mathbb{E}}$ based on the Kripke frames, i.e. the frames in which every subset is internal.

We make use of elements of point-set topology, specifically the notions of open and closed sets, base for a topology, continuous function, compact set/space and Hausdorff separation. In the later parts of the paper it is assumed that the reader has some familiarity with the basics of category theory, including the notion of a natural transformation between functors.

\section{Frames}

To establish notation, we begin by reviewing the basic theory of "general" frames for modal logic, much of which is well known.

If $R$ is a binary relation on a set $A$, then the point-image of an element $a \in A$ is the set

$$
R_{a}=\{b \in A: a R b\}
$$


Associated with $R$ are operators $\langle R\rangle$ and $[R]$ on subsets $X$ of $A$, defined by

$$
\begin{aligned}
& \langle R\rangle X=\left\{a \in A: R_{a} \cap X \neq \emptyset\right\}, \\
& {[R] X=\left\{a \in A: R_{a} \subseteq X\right\} .}
\end{aligned}
$$

A frame is a structure $\left(A, R^{A}, \beta^{A}\right)$ with $R^{A}$ a binary relation on $A$ and $\beta^{A}$ a collection of subsets of $A$ that is closed under the Boolean set operations and under the operator $\left\langle R^{A}\right\rangle$. The members of $\beta^{A}$ are the internal sets of the frame. We typically name a frame by referring to its underlying set $A$.

The operator $\left\langle R^{A}\right\rangle$ is finitely additive on $\beta^{A}$, so $\left(\beta^{A}, \cap,-, \emptyset, A,\left\langle R^{A}\right\rangle\right)$ is a modal algebra. Note that $\beta^{A}$ is closed under $\left[R^{A}\right]$, as $\left[R^{A}\right] X=-\left\langle R^{A}\right\rangle-X$.

Since $\beta^{A}$ is closed under finite intersections, it is the base of a topology $T^{A}$ on $A$ whose open sets are the unions of subsets of $\beta^{A}$. Since $\beta^{A}$ is closed under complementation, all internal sets are clopen (closed and open) in this topology, but a clopen set need not be internal (see below). We will say that the frame $A$ has a certain topological property (e.g. Hausdorff, compact) if the space $\left(A, T^{A}\right)$ has this property. A frame is image-finite if every pointimage $R_{a}^{A}$ is finite, and is image-compact if each $R_{a}^{A}$ is a compact subset of the space $\left(A, T^{A}\right)$. An image-closed frame is one in which every point-image $R_{a}^{A}$ is $T^{A}$-closed. That means that each $b \notin R_{a}^{A}$ has a basic open neighbourhood $X \in \beta^{A}$ that is disjoint from $R_{a}^{A}$. Equivalently, image-closure means that

$$
\text { if } \forall X \in \beta^{A}\left(b \in X \text { implies } a \in\left\langle R^{A}\right\rangle X\right) \text {, then } a R^{A} b \text {. }
$$

Note that the converse of this implication is always true.

A descriptive frame is one that is compact, Hausdorff and image-closed. Since a closed subset of a compact space is always compact, we see that a descriptive frame is image-compact.

A frame is full when every subset of $A$ is internal, i.e. when $\beta^{A}$ is equal to the powerset $\mathcal{P} A$ of $A$. A Kripke frame is a structure of the form $\left(A, R^{A}\right)$, identifiable with the full frame $\left(A, R^{A}, \mathcal{P} A\right)$. A discrete frame is one in which $T^{A}=\mathcal{P} A$, in which case every subset of $A$ is clopen. This holds iff each singleton $\{a\}$ is open, and hence is equivalent to requiring that each singleton belongs to $\beta^{A}$ (since an open singleton must be basic open). It follows readily that a discrete frame is always Hausdorff and image-closed.

A discrete frame need not be full; for instance if $\beta^{\omega}$ is the set of finite or cofinite subsets of $\omega=\{0,1,2, \ldots\}$, then $\left(\omega,>, \beta^{\omega}\right)$ is a discrete frame that is not full. By constructing a "one-point compactification" of it, we obtain an example of a compact Hausdorff frame that is not descriptive. This is the frame $\left(\omega+1,>, \beta^{\omega+1}\right)$, where $\omega+1$ is the ordinal $\omega \cup\{\omega\}$ and $\beta^{\omega+1}$ consists of all finite subsets of $\omega$ and their complements in $\omega+1$. The 
resulting topological space space is Hausdorff, and is also compact because any open neighbourhood of the point $\omega$ in $\omega+1$ contains all but finitely many members of $\omega$, so any open cover of the space can be reduced to a finite subcover. However the frame is not image-closed and therefore not descriptive, since the >-image of the point $\omega$ is $\{0,1,2, \ldots\}$ which is not closed, because its complement $\{\omega\}$ is not in $\beta^{\omega+1}$ and is therefore not open.

Each frame $\left(A, R^{A}, \beta^{A}\right)$ has an ultrafilter enlargement, which is a frame

$$
\left(E A, R^{E A}, \beta^{E A}\right),
$$

based on the set $E A$ of ultrafilters of the Boolean algebra $\beta^{A}$. For all $p, q \in$ $E A$, this has

$$
\begin{aligned}
& p R^{E A} q \quad \text { iff } \quad X \in q \text { implies } \quad\left\langle R^{A}\right\rangle X \in p \\
& \text { iff } \quad\left[R^{A}\right] X \in p \text { implies } \quad X \in q, \quad \text { all } X \in \beta^{A},
\end{aligned}
$$

while $\beta^{E A}=\left\{X^{E A}: X \in \beta^{A}\right\}$, where $X^{E A}=\{p \in E A: X \in p\}$. These definitions make $E A$ into a descriptive frame [6, Section 1.10].

The assignment $X \mapsto X^{E A}$ gives an isomorphism from the modal algebra $\left(\beta^{A},\left\langle R^{A}\right\rangle\right)$ of $A$ onto the modal algebra $\left(\beta^{E A},\left\langle R^{E A}\right\rangle\right)$ of $E A[6,1.10 .3]$. In particular, for each $X \in \beta^{A}$ we have

$$
\left(\left\langle R^{A}\right\rangle X\right)^{E A}=\left\langle R^{E A}\right\rangle\left(X^{E A}\right) .
$$

The definition of $E A$ is an instance of the general construction from any modal algebra $\mathfrak{A}$ of a descriptive frame $\mathfrak{A}_{+}$whose points are the ultrafilters of $\mathfrak{A}[6,1.10 .1]$. Indeed $E A$ is precisely $\left(\beta^{A},\left\langle R^{A}\right\rangle\right)_{+}$.

There is an evident insertion map $\eta_{A}: A \rightarrow E A$, having

$$
\eta_{A}(a)=\left\{X \in \beta^{A}: a \in X\right\} .
$$

Note that in general

$$
\eta_{A}(a) R^{E A} \eta_{A}(b) \quad \text { iff } \quad \forall X \in \beta^{A}\left(b \in X \text { implies } a \in\left\langle R^{A}\right\rangle X\right) .
$$

From this it follows that $a R^{A} b$ implies $\eta_{A}(a) R^{E A} \eta_{A}(b)$, and that

$$
A \text { is image-closed iff } \quad \eta_{A}(a) R^{E A} \eta_{A}(b) \text { implies } a R^{A} b .
$$

If $A$ is Hausdorff, then $\eta_{A}$ is injective since distinct points $a, b$ then have disjoint basic open neighbourhoods, so $\eta_{A}(a) \neq \eta_{A}(b)$. Conversely, injectivity of $\eta_{A}$ implies that $A$ is Hausdorff (this uses the closure of $\beta^{A}$ under complements). If $A$ is compact, then $\eta_{A}$ is surjective, since then each ultrafilter $p$ of 
$\beta^{A}$ has nonempty intersection, and any $a \in \bigcap p$ has $p=\eta_{A}(a)$. Conversely it can be shown that surjectivity of $\eta_{A}$ implies that $A$ is compact.

Thus when $A$ is compact and Hausdorff, $\eta_{A}$ is a bijection. Its inverse $\varepsilon_{A}: E A \rightarrow A$ assigns to each $p \in E A$ the unique point in its intersection, so that $\bigcap p=\left\{\varepsilon_{A} p\right\}$. Thus for any $X \in \beta^{A}, X \in p$ iff $\varepsilon_{A}(p) \in X$.

Later we will need to understand the nature of $\varepsilon_{A}$ when $A$ is itself some ultrafilter enlargement $E C$. Then $\varepsilon_{E C}(p) \in E C$ and for each internal set $X$ of the frame $C, X \in \varepsilon_{E C}(p)$ iff $\varepsilon_{E C}(p) \in X^{E C}$. Therefore from the given description of $\varepsilon_{A}$,

$$
\varepsilon_{E C}(p)=\left\{X \in \beta^{C}: X^{E C} \in p\right\}
$$

\section{Modal Maps}

We will study the following properties of a function $f: A \rightarrow B$ between two frames.

- $f$ is internal if $X \in \beta^{B}$ implies $f^{-1} X \in \beta^{A}$, i.e. $f^{-1}$ preserves internal sets.

- $f$ has the forth property if, for each $X \in \beta^{B},\left\langle R^{A}\right\rangle f^{-1} X \subseteq f^{-1}\left\langle R^{B}\right\rangle X$, which means that for each $a \in A$,

$$
\exists b\left(a R^{A} b \& f(b) \in X\right) \text { implies } f(a) \in\left\langle R^{B}\right\rangle X .
$$

- $f$ has the strong forth property if, for all $a, b \in A$,

$$
a R^{A} b \text { implies } f(a) R^{B} f(b) .
$$

- $f$ has the back property if, for each $X \in \beta^{B}, f^{-1}\left\langle R^{B}\right\rangle X \subseteq\left\langle R^{A}\right\rangle f^{-1} X$, which means that for each $a \in A$,

$$
f(a) \in\left\langle R^{B}\right\rangle X \quad \text { implies } \exists b\left(a R^{A} b \& f(b) \in X\right) .
$$

- $f$ has the strong back property if, for all $a \in A$ and $c \in B$,

$$
f(a) R^{B} c \text { implies } \exists b\left(a R^{A} b \& f(b)=c\right) .
$$

- $f$ is a modal map if it is internal and has the back and forth properties.

- $f$ is a bounded morphism if it is internal and has the strong back and strong forth properties. 
An internal function is topologically continuous, i.e. $f^{-1} X$ is $T^{A}$-open for all $T^{B}$-open $X$. But a continuous function need not be internal. For example, if $\left(\omega,>, \beta^{\omega}\right)$ is the frame described above, while $\{0,1\}$ is regarded as a full frame under any binary relation and $f: \omega \rightarrow\{0,1\}$ has $f(n)=1$ iff $n$ is even, then $f$ is continuous, since the topology on $\omega$ is discrete, but is not internal, since $f^{-1}\{1\} \notin \beta^{\omega}$. Notice also that $f^{-1}\{1\}$ is clopen here but not internal.

It is easy to see that the strong forth/strong back property implies the corresponding forth/back property, so a bounded morphism is a modal map. Here are some further relationships between these properties:

\section{THEOREM 1.}

(1) If $f: A \rightarrow B$ has the forth property and $B$ is image-closed, then $f$ has the strong forth property.

(2) If $f: A \rightarrow B$ has the back property, and $B$ is discrete, then $f$ has the strong back property.

(3) If $f: A \rightarrow B$ is continuous and has the back property, then $f$ has the strong back property provided that $B$ is Hausdorff and $A$ is imagecompact.

(4) If $B$ is discrete, then any modal map $f: A \rightarrow B$ is a bounded morphism.

(5) Any modal map from an image-compact frame to an image-closed Hausdorff frame is a bounded morphism. In particular, any modal map between descriptive frames is a bounded morphism.

PROOF.

(1) Let $a R^{A} b$. We want $f(a) R^{B} f(b)$. By image-closure it is enough to show that if $f(b) \in X \in \beta^{B}$, then $f(a) \in\left\langle R^{B}\right\rangle X$. But that is immediate from the forth property.

(2) Let $f(a) R^{B} c$. Then $f(a) \in\left\langle R^{B}\right\rangle\{c\}$. But if $B$ is discrete, then $\{c\} \in \beta^{B}$ and so by the back property there exists $b \in A$ with $a R^{A} b$ and $f(b) \in\{c\}$, i.e. $f(b)=c$ as required.

(3) Suppose $f$ is continuous with the back property, $B$ is Hausdorff and $A$ is image-compact. Let $f(a) R^{B} c$. Suppose, for the sake of contradiction, that there is no $b \in A$ with $a R^{A} b$ and $f(b)=c$. Then for each $b \in R_{a}^{A}$, since $B$ is Hausdorff there are disjoint sets $X_{b}, Y_{b} \in \beta^{B}$ with $c \in X_{b}$ 
and $f(b) \in Y_{b}$. Since $f$ is continuous, $\left\{f^{-1} Y_{b}: b \in R^{A} a\right\}$ is then a cover of $R_{a}^{A}$ by open sets. But $R_{a}^{A}$ is compact by assumption, so for some $n$ there exist $b_{1}, \ldots, b_{n}$ such that $\left\{f^{-1} Y_{b_{1}}, \ldots, f^{-1} Y_{b_{n}}\right\}$ is a cover of $R_{a}^{A}$. Now let $X=X_{b_{1}} \cap \cdots \cap X_{b_{n}} \in \beta^{B}$. Then $c \in X$, and so as $f(a) R^{B} c$ we get $f(a) \in\left\langle R^{B}\right\rangle X$. But now if $a R^{A} b$, then $f(b) \in Y_{b_{i}}$ for some $i$, hence $f(b) \notin X_{b_{i}}$ and so $f(b) \notin X$. Thus $X$ contradicts the back property.

(4) From (1) and (2), since any discrete frame is image-closed.

(5) From (1) and (3), and the fact that descriptive frames are image-closed, Hausdorff and image-compact.

An internal function $f: A \rightarrow B$ induces the Boolean algebra homomorphism $f_{+}: \beta^{B} \rightarrow \beta^{A}$ where $f_{+}(X)=f^{-1} X$. Thus a modal map has $f_{+}\left(\left\langle R^{B}\right\rangle X\right)=\left\langle R^{A}\right\rangle f_{+}(X)$, which is precisely the condition for $f_{+}$to be a modal algebra homomorphism from $\left(\beta^{B},\left\langle R^{B}\right\rangle\right)$ to $\left(\beta^{A},\left\langle R^{A}\right\rangle\right)$. But such a homomorphism induces a map $E A \rightarrow E B$ that is a bounded morphism and which acts by pulling ultrafilters of $\beta^{A}$ back along $f_{+}$to ultrafilters of $\beta^{B}[6$, 1.10.9]. The upshot is that any modal map $f: A \rightarrow B$ induces a bounded morphism $E f: E A \rightarrow E B$ given by

$$
E f(p)=\left\{X \in \beta^{B}: f^{-1} X \in p\right\} .
$$

It is readily checked that the back and forth properties are preserved by composition of functions, and that the composition of modal maps is a modal map. Hence there is a category $\mathbf{F m}$ whose objects are the frames and whose arrows are the modal maps, with identity arrows being the identity functions and composition of arrows being their functional composition. Let DFm be the full subcategory of $\mathbf{F m}$ whose objects are the descriptive frames. The term "full" here means that the arrows in the subcategory comprise all the arrows in the larger category whose domain and codomain are objects in the subcategory. By Theorem 1(5), the arrows of DFm are all the bounded morphisms between descriptive frames. The assignments $A \mapsto E A$ and $f \mapsto E f$ define a functor $E: \mathbf{F m} \rightarrow \mathbf{F m}$ that maps $\mathbf{F m}$ into $\mathbf{D f m}$.

A modal map $f: A \rightarrow B$ is an isomorphism when it has an inverse in $\mathbf{F m}$, i.e. a modal map $g: B \rightarrow A$ having $g \circ f=1_{A}$ and $f \circ g=1_{B}$, where "1" denotes an identity arrow. This implies that $f$ is bijective and $g$ is its set-theoretic inverse. We write $A \cong B$ as usual to indicate that there is an isomorphism from $A$ to $B$. In general, a modal map is an isomorphism in 
Fm precisely when it is bijective as a set function and its inverse function is also a modal map.

An isomorphism is a homeomorphism and so preserves any purely topological properties. Thus a frame isomorphic to a Hausdorff/compact frame is also Hausdorff/compact. But frame isomorphism does not preserve the property of being image-closed. Instead each frame has an "image-closure" in the following sense.

\section{THEOREM 2.}

(1) For each frame $A$ there is an an image-closed frame $A^{*}$ and an isomorphism $\sigma_{A}: A \rightarrow A^{*}$ in $\boldsymbol{F m}$ such that $A$ and $A^{*}$ have the same underlying set and internal subsets, with $A^{*}=A$ iff $A$ is image-closed. Moreover, $A$ and $A^{*}$ have the same ultrafilter enlargement, i.e. $E A=E\left(A^{*}\right)$, and $\eta_{A}=\eta_{A^{*}} \circ \sigma_{A}$ :

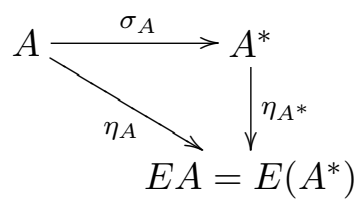

(2) If $E A=E B$, then $A^{*}=B^{*}$. Hence $A^{*}$ is the only image-closed frame having the same ultrafilter enlargement as $A$.

(3) For each modal map $f: A \rightarrow B$ there is a modal map $f^{*}: A^{*} \rightarrow B^{*}$ that is the unique such map making the following diagram commute.

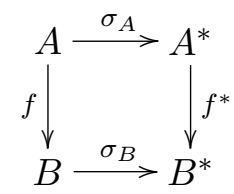

Moreover, $\left(\eta_{A}\right)^{*}=\eta_{A^{*}}$.

(4) Any compact Hausdorff frame is isomorphic to a descriptive frame with the same underlying set and internal subsets.

ProOF.

(1) Given frame $\left(A, R^{A}, \beta^{A}\right)$, let $\left(A^{*}, R^{A^{*}}, \beta^{A^{*}}\right)$ have $A^{*}=A, \beta^{A^{*}}=\beta^{A}$, and

$$
a R^{A^{*}} b \text { iff } \forall X \in \beta^{A}\left(b \in X \text { implies } a \in\left\langle R^{A}\right\rangle X\right) .
$$


Then $R^{A} \subseteq R^{A^{*}}$, and so $\left\langle R^{A}\right\rangle X \subseteq\left\langle R^{A^{*}}\right\rangle X$. But if $a R^{A^{*}} b \in X \in \beta^{A}$, then $a \in\left\langle R^{A}\right\rangle X$, showing that $\left\langle R^{A^{*}}\right\rangle X \subseteq\left\langle R^{A}\right\rangle X$ and hence $\left\langle R^{A^{*}}\right\rangle X=$ $\left\langle R^{A}\right\rangle X$, for all $X \in \beta^{A}$. Thus $A^{*}$ is indeed a frame as defined, and is image-closed by the definition of $R^{A^{*}}$. If $A$ itself is image-closed then $a R^{A^{*}} b$ implies $a R^{A} b$, so $A^{*}=A$.

The identity function on $A$ is a bijective modal map $\sigma_{A}: A \rightarrow A^{*}$, whose inverse is a modal map $\sigma_{A}^{-1}: A^{*} \rightarrow A$. Hence $\sigma_{A}$ is an isomorphism $A \cong A^{*}$. In fact $\sigma_{A}$ has the strong forth property as $R^{A} \subseteq R^{A^{*}}$, but has the strong back property iff $R^{A}=R^{A^{*}}$ iff $A$ is image-closed. Likewise $\sigma_{A}^{-1}$ has the strong back property but has the strong forth property iff $A=A^{*}$.

Since the modal algebras $\left(\beta^{A},\left\langle R^{A}\right\rangle\right)$ and $\left(\beta^{A^{*}},\left\langle R^{A^{*}}\right\rangle\right)$ are identical, so too are the frames $E A$ and $E\left(A^{*}\right)$. That $\eta_{A}=\eta_{A^{*}} \circ \sigma_{A}$ follows because $\eta_{A}$ and $\eta_{A^{*}}$ are the same functions.

(2) Let $C$ and $D$ be any image-closed frames that have the same ultrafilter enlargement. We show that $C$ and $D$ are identical. Firstly, their underlying sets must be equal, for if $a \in C-D$, say, then $D \notin \eta_{C}(a) \in E C$, hence $\eta_{C}(a) \notin E D$, contradicting the assumption $E C=E D$. Next their collections of internal subsets must be equal, for if $X \in \beta^{C}-\beta^{D}$, say, then $X \neq \emptyset$ and any $a \in X$ gives $X \in \eta_{C}(a)$, so $\eta_{C}(a) \nsubseteq \beta^{D}$ and hence $\eta_{C}(a) \notin E D$, again contradicting $E C=E D$. Now we have $\left(C, \beta^{C}\right)=\left(D, \beta^{D}\right)$, so the insertion maps $\eta_{C}$ and $\eta_{D}$ are identical. But since $C$ and $D$ are image-closed, we have $\left[a R^{C} b\right.$ iff $\left.\eta_{C}(a) R^{E C} \eta_{C}(b)\right]$ and $\left[a R^{D} b\right.$ iff $\left.\eta_{D}(a) R^{E D} \eta_{D}(b)\right]$ in general, so as $R^{E C}=R^{E D}$ and $\eta_{C}=\eta_{D}$, it follows that $R^{C}=R^{D}$. Hence $C$ and $D$ are indeed identical.

Now applying this result: if $A$ and $B$ are any frames with $E A=E B$, then by (1), $E\left(A^{*}\right)=E\left(B^{*}\right)$, and so $A^{*}=B^{*}$ as both are image closed. If further $B$ is image closed, then $B^{*}=B$ so $A^{*}=B$.

(3) Given $f: A \rightarrow B$, let $f^{*}$ be the same function as $f$ but with domain and codomain being $A^{*}$ and $B^{*}$ instead of $A$ and $B$ respectively. Since $\sigma_{A}$ and $\sigma_{B}$ are identity functions, this is the only way to define $f^{*}$ to make the diagram in (3) commute.

But $\left\langle R^{A}\right\rangle f^{-1} X=f^{-1}\left\langle R^{B}\right\rangle X$ iff $\left\langle R^{A^{*}}\right\rangle f^{-1} X=f^{-1}\left\langle R^{B^{*}}\right\rangle X$ because $\left\langle R^{A^{*}}\right\rangle=\left\langle R^{A}\right\rangle$ and $\left\langle R^{B^{*}}\right\rangle=\left\langle R^{B}\right\rangle$, so $f$ is a modal map iff $f^{*}$ is.

When $B=E A$ then $\sigma_{B}$ is just the identity arrow on $E A$, as $E A=(E A)^{*}$ because $E A$ is image-closed. Hence when $f$ is $\eta_{A}$, the square of (3) is just the triangle of (1), showing that $\left(\eta_{A}\right)^{*}=\eta_{A^{*}}$. 
(4) If $A$ is compact and Hausdorff, then $A^{*}$ is compact and Hausdorff and image-closed.

Note that since $A^{*}$ is image-closed, $\left(A^{*}\right)^{*}=A^{*}$ with $\sigma_{A^{*}}=1_{A^{*}}$. Also, as noted in the proofs, since $E A$ is image-closed for any $A$ we have $(E A)^{*}=$ $E A=E\left(A^{*}\right)$, so we could write $E A^{*}$ for that structure with impunity.

If $A$ is the compact Hausdorff frame $\left(\omega+1,>, \beta^{\omega+1}\right)$ discussed above, then $A \cong A^{*}$ but $A$ and $A^{*}$ are distinct as $A$ is not image-closed while $A^{*}$ is, and so $A^{*}$ is descriptive. This is a delicate example: the only difference between $A$ and $A^{*}$ here is that the point $\omega$ has $\omega R^{A^{*}} \omega$ but not $\omega R^{A} \omega$.

\section{Some Equivalent Categories}

Let $\mathbf{C H F m}$ be the full subcategory of $\mathbf{F m}$ whose objects are the compact Hausdorff frames. DFm is in turn a full subcategory of CHFm but unlike in DFm, not all arrows in CHFm are bounded morphisms. This is shown by the example just considered: the identity function on $\omega+1$ is a CHFmarrow $\omega+1 \rightarrow(\omega+1)^{*}$ that is does not have the strong back property.

The categories CHFm and DFm are equivalent. In general, an equivalence between categories $\mathbf{C}$ and $\mathbf{D}$ is given by a pair of functors $F: \mathbf{C} \rightarrow \mathbf{D}$ and $G: \mathbf{D} \rightarrow \mathbf{C}$ such that $G \circ F$ is naturally isomorphic to the identity functor $1_{\mathbf{C}}$ on $\mathbf{C}$ and $F \circ G$ is naturally isomorphic to $1_{\mathbf{D}}$. This implies that each C-object $A$ is isomorphic to $G(F(A))$ and each $\mathbf{D}$-object $B$ is isomorphic to $F(G(B))$. This notion of equivalence gives an equivalence relation between categories. If more strongly $G \circ F=1_{\mathbf{C}}$ and $F \circ G=1_{\mathbf{D}}$, then $F$ and $G$ make $\mathbf{C}$ and $\mathbf{D}$ isomorphic.

If $\mathbf{C}$ is a full subcategory of $\mathbf{D}$ and every $\mathbf{D}$-object is isomorphic to a C-object, then $\mathbf{C}$ and $\mathbf{D}$ are equivalent [8, IV.4]. In the case of $\mathbf{C H F m}$ and DFm we can see this explicitly. Let $F: \mathbf{C H F m} \rightarrow \mathbf{D F m}$ be given by the constructions of Theorem 2, i.e. $F A=A^{*}$ and $F f=f^{*}$, while $G: \mathbf{D F m} \rightarrow \mathbf{C H F m}$ is the inclusion functor: $G A=A$ and $G f=f$. For any modal map $f: A \rightarrow B$ the commuting of the diagram

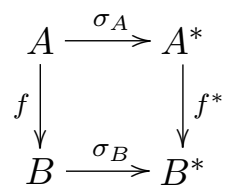


of Theorem 2(3) means that the isomorphisms $\sigma_{A}$ are natural in $A$ and shows that this $F$ and $G$ give an equivalence between CHFm and DFm. Since $A=A^{*}$ when $A$ is descriptive, we in fact have $F \circ G=1_{\mathbf{D F m}}$.

Our view of this equivalence can be refined by considering the category of all ultrafilter enlargements of frames. This is the full subcategory UEFm of $\mathbf{C H F m}$ based on the collection of objects $\{E A: A$ in $\mathbf{F m}\}$. It is shown in the next theorem that each compact Hausdorff $A$ is isomorphic to $E A$, implying that UEFm is equivalent to CHFm. But UEFm is a full subcategory of $\mathbf{D F m}$ and hence equivalent to $\mathbf{D F m}$ for the same reason. Since $E\left(A^{*}\right)=E A$ in general, the equivalences between the three categories commute appropriately.

The distinction between $\mathbf{C H F m}$ and $\mathbf{D F m}$ is more significant than that between DFm and UEFm. For any compact Hausdorff $A$ we have isomorphisms

$$
A \cong A^{*} \cong E A=E\left(A^{*}\right)
$$

If $A$ is not image-closed, hence not in DFm, then the first isomorphism is not a bounded morphism. But the second isomorphism, between the descriptive frames $A^{*}$ and $E A$, is always a bounded morphism, as Theorem 3 will show. In general, if $B$ is descriptive, then $B$ and $E B$ are indistinguishable frametheoretically as well as categorically, and may be thought of a relabellings of each other. Another perspective is that DFm is the closure of UEFm under isomorphism in the category of frames and bounded morphisms, whereas CHFm is the closure of UEFm under isomorphism in the category of frames and modal maps. Note that the frame $(\omega+1)^{*}$ is in DFm but not UEFm, since its points are not ultrafilters of some frame.

Category $\mathbf{C}$ is called dual to category $\mathbf{D}$ if it is equivalent to the opposite category $\mathbf{D}^{o p}$ of $\mathbf{D} . \mathbf{D}^{o p}$ has the same objects as $\mathbf{D}$, while the $\mathbf{D}^{o p}$-arrows $A \rightarrow B$ are the $\mathbf{D}$-arrows $B \rightarrow A$. A duality can alternatively be described as a pair of functors between $\mathbf{C}$ and $\mathbf{D}$ that are contravariant (i.e. reverse the direction of arrows) and whose composites are naturally isomorphic to the identity functors on $\mathbf{C}$ and $\mathbf{D}$. It was shown in [5] that the category MA of modal algebras and homomorphisms is dual to DFm. Hence MA is dual to CHFm and UEFm as well.

\section{Insertions Are Modal}

If we were to focus on categories of frames whose arrows were all bounded morphisms, then many insertion maps $\eta_{A}: A \rightarrow E A$ would be missing as they are not bounded. This is the ultimate reason why we need to consider 
the more general notion of a modal map in order to understand the categorical nature of these structures properly. Here are the main properties of insertions.

Theorem 3. For any frame A:

(1) $\eta_{A}$ is a modal map with the strong forth property.

(2) $\eta_{A}$ is a bounded morphism iff $A$ is image-compact.

(3) If $A$ is image-finite, then $\eta_{A}$ is a bounded morphism.

(4) If $A$ is discrete, then $\eta_{A}$ is a bounded morphism iff $A$ is image-finite.

(5) If $A$ is compact and Hausdorff, then $\eta_{A}$ is an isomorphism $A \cong E A$ in $\mathbf{C H F m}$ with inverse $\varepsilon_{A}: E A \cong A$. In this case $\varepsilon_{A}$ is a bounded morphism iff $\eta_{A}$ is a bounded morphism iff $A$ is descriptive.

Proof.

(1) First, $\eta_{A}$ is internal, since if $X^{E A} \in \beta^{E A}$, then $\eta_{A}^{-1} X^{E A}=X \in \beta^{A}$. For the strong forth property, we have already noted that if $a R^{A} b$ then $\eta_{A}(a) R^{E A} \eta_{A}(b)$. Finally, $\eta_{A}$ has the back property, since for all $X^{E A} \in$ $\beta^{E A}$, if $\eta_{A}(a) \in\left\langle R^{E A}\right\rangle\left(X^{E A}\right)=\left(\left\langle R^{A}\right\rangle X\right)^{E A}$, then $a \in\left\langle R^{A}\right\rangle X$, so there exists $b$ with $a R^{A} b$ and $b \in X$, hence $\eta_{A}(b) \in X^{E A}$.

(2) If $A$ is image-compact, then it follows by (1) and Theorem 1(5) that $\eta_{A}$ is a bounded morphism since $E A$ is image-closed and Hausdorff.

For the converse, suppose that $A$ is not image-compact. Then there is some point-image $R_{a}^{A}$ that has an open cover $\mathcal{C}$ with no finite subcover. We can assume that the members of $\mathcal{C}$ are basic open, so $\mathcal{C} \subseteq \beta^{A}$. Put

$$
q_{0}=\left\{X \in \beta^{A}:\left[R^{A}\right] X \in \eta_{A}(a)\right\} \cup\{-X: X \in \mathcal{C}\} \subseteq \beta^{A} .
$$

Now for any $X_{1}, \ldots, X_{n} \in \mathcal{C}$ there exists some $b \in R_{a}^{A}-\left(X_{1} \cup \cdots X_{n}\right)$, so then

$$
b \in \bigcap\left\{X \in \beta^{A}:\left[R^{A}\right] X \in \eta_{A}(a)\right\} \cap-X_{1} \cap \cdots \cap-X_{n} .
$$

This shows that $q_{0}$ has the finite intersection property and hence extends to a $\beta^{A}$-ultrafilter $q \in E A$. Then $\eta_{A}(a) R^{E A} q$ by definition of $q_{0}$. But for any $b \in R_{a}^{A}$ we have $\eta_{A}(b) \neq q$ because there is some $X \in \mathcal{C}$ with $b \in X$, hence $X \in \eta_{A}(b)$, while $-X \in q$. Thus $\eta_{A}$ does not have the strong back property. 
(3) From (2), as image-finiteness implies image-compactness.

(4) From (2), as in a discrete space a set is compact iff it is finite.

(5) Let $A$ be compact and Hausdorff. We noted already that in this case $\eta_{A}$ is a bijection with set inverse $\varepsilon_{A}$. Then $\varepsilon_{A}$ is also internal, since $\varepsilon_{A}^{-1} X=X^{E A} \in \beta^{E A}$ for $X \in \beta^{A}$. Since by (1) $\eta_{A}$ has the strong forth property, it follows that $\varepsilon_{A}$ has the strong back property. Also $\varepsilon_{A}$ has the forth property, since if $p R^{E A} q$ and $\varepsilon_{A}(q) \in X \in \beta^{A}$, then $X \in q$ and hence $\left\langle R^{A}\right\rangle X \in p$, implying $\varepsilon_{A}(p) \in\left\langle R^{A}\right\rangle X$. Thus $\varepsilon_{A}$ is a modal map providing an inverse for $\eta_{A}$ in CHFm.

Finally, the strong forth property holds for $\varepsilon_{A}$ iff the strong back property holds for $\eta_{A}$ iff $\left[\eta_{A}(a) R^{E A} \eta_{A}(b)\right.$ implies $\left.a R^{A} b\right]$ iff $A$ is image-closed.

Illustrating with our running example once more, in the diagram

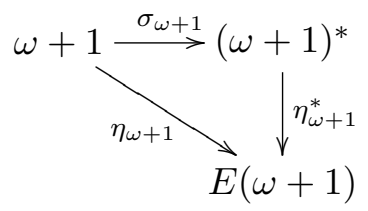

all three arrows are isomorphisms in CHFm, but only $\eta_{\omega+1}^{*}$ is a bounded morphism.

\section{Freeness of Ultrafilter Enlargements}

Each modal frame freely generates its ultrafilter enlargement in the following sense.

TheOrem 4. If $A$ is any frame and $B$ any compact Hausdorff frame, then for each modal map $f: A \rightarrow B$ there is a unique modal map $\tilde{f}: E A \rightarrow B$ that extends $f$ along $\eta_{A}$, i.e. $f=\tilde{f} \circ \eta_{A}$ :

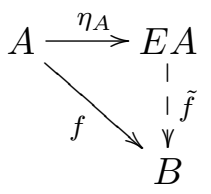

This construction has $\widetilde{f^{*}}=\widetilde{\sigma_{B} \circ f}$ and $\widetilde{\eta_{A}}=1_{E A}$. If $A$ is compact Hausdorff, then $\varepsilon_{A}=\widetilde{1_{A}}$. 
Proof. For the existence of $\tilde{f}$, given $f$ we define $\tilde{f}=E f \circ \varepsilon_{B}$ :

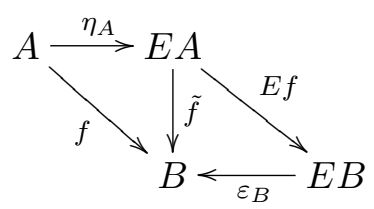

Thus $\tilde{f}$ is the composition of two modal maps, and therefore a modal map itself. (If $B$ is descriptive, then $\tilde{f}$ is a bounded morphism by Theorem 1(5).) $\tilde{f}(p)$ is the unique point in the intersection of $E f(p)$, i.e. the unique member of

$$
\bigcap\left\{X \in \beta^{B}: f^{-1} X \in p\right\} .
$$

But $f(a) \in \bigcap\left\{X \in \beta^{B}: f^{-1} X \in \eta_{A}(a)\right\}$, so $f(a)=\tilde{f}\left(\eta_{A}(a)\right)$ as required.

The uniqueness of $\tilde{f}$ is topologically determined, and does not depend on the back and forth properties: $\tilde{f}$ is the only continuous extension of $f$ along $\eta_{A}$. To see this, suppose $g: E A \rightarrow B$ is continuous with $f=g \circ \eta_{A}$. Then for any $p \in E A$, if $g(p) \in X \in \beta^{B}$, then $g^{-1} X$ contains $p$ and is open in $E A$, so $p \in Y^{E A} \subseteq g^{-1} X$ for some $Y \in \beta^{A}$. Hence

$$
f^{-1} X=\eta_{A}^{-1}\left(g^{-1} X\right) \supseteq \eta_{A}^{-1}\left(Y^{E A}\right)=Y \in p,
$$

so $f^{-1} X \in p$ (since $f^{-1} X \in \beta^{A}$ ), and therefore $\tilde{f}(p) \in X$. This shows that every basic open neighbourhood of $g(p)$ in $B$ contains $\tilde{f}(p)$. Since $B$ is Hausdorff, it follows that $g(p)=\tilde{f}(p)$.

That $\widetilde{f *}=\widetilde{\sigma_{B} \circ f}$ follows from the commuting diagram

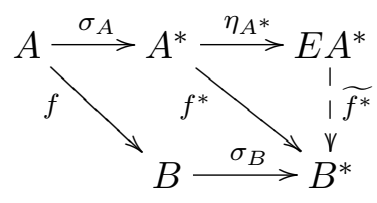

which shows that $\widetilde{f^{*}}$ is the unique extension of $\sigma_{B} \circ f$ along $\eta_{A}=\eta_{A^{*}} \circ \sigma_{A}$.

Finally, the diagrams
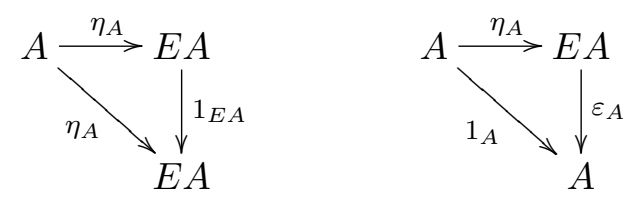

show that $\widetilde{\eta_{A}}=1_{E A}$ and, if $A$ is compact Hausdorff, then $\varepsilon_{A}=\widetilde{1_{A}}$. 
COROLlary 5. If $B$ is descriptive, then $\widetilde{f^{*}}=\tilde{f}$ for any modal map $f$ with codomain $B$.

Proof. In this case, $B=B^{*}$ and $\sigma_{B}=1_{B}$.

The construction of Theorem 4 will be applied later to a map of the form $g: B \rightarrow E C$. Then $\tilde{g}$ is the composition of

$$
E B \stackrel{E g}{\longrightarrow} E E C \stackrel{\varepsilon_{E C}}{\longrightarrow} E C
$$

so by the earlier description of $\varepsilon_{E C}$ in equation (I),

$$
\tilde{g}(q)=\varepsilon_{E C}(E g(q))=\left\{X \in \beta^{C}: X^{E C} \in E g(q)\right\},
$$

and therefore by the definition of $E g$,

$$
\tilde{g}(q)=\left\{X \in \beta^{C}: g^{-1}\left(X^{E C}\right) \in q\right\} .
$$

\section{Reflective Subcategories}

In categorical parlance [8, III.1], Theorem 4 states that $\left(E A, \eta_{A}\right)$ is a universal arrow from the $\mathbf{F m}$-object $A$ to the inclusion functor $G: \mathbf{C H F m} \rightarrow \mathbf{F m}$ defined by $G A=A$ and $G f=f$. This implies [8, IV.1] that the functor $E: \mathbf{F m} \rightarrow \mathbf{C H F m}$ is left adjoint to $G$ (and $G$ is right adjoint to $E$ ). The arrows $\eta_{A}$ for all $\mathbf{F m}$-objects $A$ are the components of a natural transformation $\eta: 1_{\mathbf{F m}} \rightarrow E$ from the identity functor on $\mathbf{F m}$ to $E$ that is called the unit of the adjunction. The counit is a natural transformation $E \rightarrow 1_{\mathrm{CHFm}}$ whose components are the arrows $\widetilde{1_{A}}$. We saw that these are just the modal maps $\varepsilon_{A}$ for all compact Hausdorff $A$.

If a category $\mathbf{C}$ is a subcategory of a category $\mathbf{D}$, and the inclusion functor $\mathbf{C} \rightarrow \mathbf{D}$ has a left adjoint $\mathbf{D} \rightarrow \mathbf{C}$, then $\mathbf{C}$ is called a reflective subcategory of $\mathbf{D}$, and the left adjoint is the reflector. So we have established that $\mathbf{C H F m}$ is a reflective subcategory of $\mathbf{F m}$ with reflector $E$. In general, if $\mathbf{C}$ is a full reflective subcategory of $\mathbf{D}$, then the components of the associated counit are always isomorphisms in $\mathbf{D}$, and their inverses are the components of the unit $[2,3.4 .1]$. In our case, we showed in Theorem 3(5) that $\varepsilon_{A}$ is an isomorphism with inverse $\eta_{A}$, on the way to establishing in Theorem 4 that $E: \mathbf{F m} \rightarrow \mathbf{C H F m}$ is a reflector.

Note that since the values of the functor $E$ actually lie in the subcategory UEFm of DFm, $E$ can also serve as a left adjoint to the inclusion $\mathbf{U E F m} \rightarrow$ $\mathbf{F m}$ or the inclusion $\mathbf{D F m} \rightarrow \mathbf{F m}$, so UEFm and $\mathbf{D F m}$ are reflective 
subcategories of $\mathbf{F m}$, and indeed all inclusions between these categories are reflective.

From the very existence of a reflective subcategory a great deal of structure flows on general categorical grounds, as will now be explained.

\section{Monads}

Any pair of adjoint functors defines a structure called a monad on the domain of the functor that is the left adjoint. Then the monad gives rise to two special categories that are related to the domain of the right adjoint, one due to Eilenberg and Moore [4] and the other to Kleisli [7]. This general theory is available in several references, including [8, Chapter VI], [1, Chapter 3] and $[3$, Chapter 4$]$.

In our frame-theoretic case of the adjoint pair $(E, G)$, description of the monad is simplified because $G$ is an inclusion functor that can be notationally suppressed. The monad then is the triple $\mathbb{E}=(E, \eta, \mu)$, where the functor $E: \mathbf{F m} \rightarrow \mathbf{F m}$ and the natural transformation $\eta: 1_{\mathbf{F m}} \rightarrow E$ have already been defined, and $\mu$ is the natural transformation from $E \circ E$ to $E$ whose components $\mu_{A}: E E A \rightarrow E A$ are the bounded morphisms $\varepsilon_{E A}$ for all frames $A$. The definition of "monad" stipulates that for each $A$ the following diagrams commute:
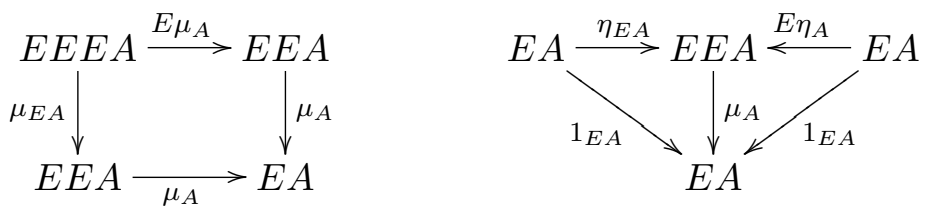

(mon)

An $\mathbb{E}$-algebra is, by definition, a pair $(A, \xi)$ comprising an $\mathbf{F m}$-object $A$ and an $\mathbf{F m}$-arrow $\xi: E A \rightarrow A$, called the structure map of the algebra, such that the following commute:
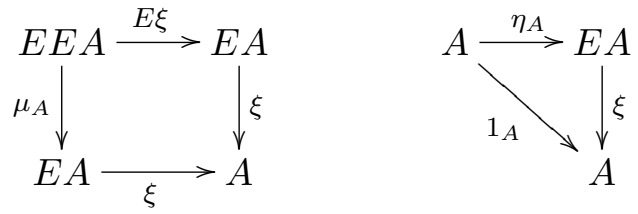

For example, by the square and the left triangle in (mon), the pair $\left(E A, \mu_{A}\right)$ is an $\mathbb{E}$-algebra, known as the free $\mathbb{E}$-algebra on $A$. 
A morphism $f:(A, \xi) \rightarrow\left(A^{\prime}, \xi^{\prime}\right)$ of $\mathbb{E}$-algebras is a Fm-arrow $f: A \rightarrow A^{\prime}$ for which the following square commutes:

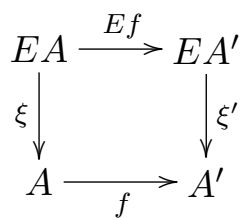

For example, by the square in (alg), the structure map $\xi$ is itself a morphism from the free algebra $\left(E A, \mu_{A}\right)$ to $(A, \xi)$.

For any monad $\mathbb{E}$ on $\mathbf{F m}$, the $\mathbb{E}$-algebras and their morphisms form the Eilenberg-Moore category $\mathbf{F m}^{\mathbb{E}}$ of $\mathbb{E}$. There is a "forgetful" functor $G^{\mathbb{E}}: \mathbf{F m}^{\mathbb{E}} \rightarrow \mathbf{F m}$ taking the algebra $(A, \xi)$ to its underlying object $A$, and a morphism $f$ to itself as an $\mathbf{F m}$-arrow. $G^{\mathbb{E}}$ has a left adjoint $F^{\mathbb{E}}: \mathbf{F m} \rightarrow \mathbf{F m}^{\mathbb{E}}$ that on objects has $F^{\mathbb{E}} A=\left(E A, \mu_{A}\right)=$ the free $\mathbb{E}$-algebra on $A$, and on arrows has $F^{\mathbb{E}} f=E f$. The adjoint pair $\left(F^{\mathbb{E}}, G^{\mathbb{E}}\right)$ defines a monad on $\mathbf{F m}$ which is just the original monad $\mathbb{E}$ - this was the motivating purpose of the Eilenberg-Moore construction: to show that any monad is defined by an adjoint pair.

Given $\mathbb{E}$ as the monad defined by an adjoint pair $(E, G)$, the connection between $\mathbf{F m}^{\mathbb{E}}$ and the domain of the right adjoint $G: \mathbf{C H F m} \rightarrow \mathbf{F m}$ is provided by a comparison functor $K: \mathbf{C H F m} \rightarrow \mathbf{F m}^{\mathbb{E}}$ that in our frame case has $K A=\left(A, \varepsilon_{A}\right)$ and for a CHFm-arrow $f: A \rightarrow B$ has $K f=f$ : $\left(A, \varepsilon_{A}\right) \rightarrow\left(B, \varepsilon_{B}\right)$. That every $\mathbf{C H F m}$-arrow $f$ becomes a morphism of $\mathbb{E}$-algebras in this way is seen from the diagram

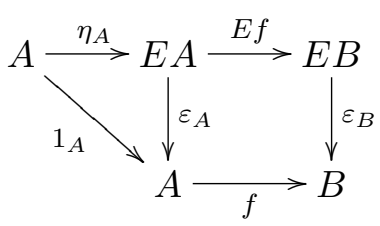

This indicates that $f \circ \varepsilon_{A}$ extends $f$ along $\eta_{A}$, so is equal to $\tilde{f}=\varepsilon_{B} \circ E f$, and hence the square commutes as desired. $K$ is the unique functor for which $G^{\mathbb{E}} \circ K=G$ and $K \circ E=F^{\mathbb{E}}$ :

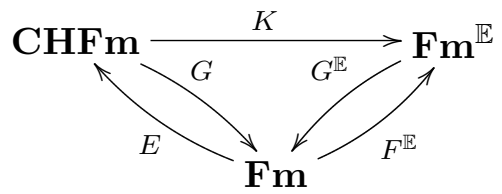

It is evident that $K$ acts injectively on objects and bijectively on arrows. In fact it is bijective on objects too, and so 
$K$ is an isomorphism between $\mathbf{C H F m}$ and $\mathbf{F} \mathbf{m}^{\mathbb{E}}$.

This is because for every $\mathbb{E}$-algebra $(A, \xi)$, the structure map $\xi$ is an isomorphism with inverse $\eta_{A}$ (cf. $[3,4.2 .3,4.2 .4]$ ). To see why, observe that since $\xi \circ \eta_{A}=1_{A}$ by (alg), we get $\eta_{A} \circ \xi \circ \eta_{A}=\eta_{A}$, so the diagram

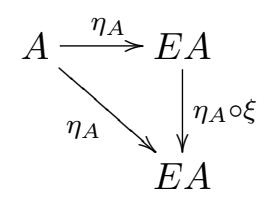

commutes, showing that $\eta_{A} \circ \xi=\widetilde{\eta_{A}}=1_{E A}$. Thus $\xi$ and $\eta_{A}$ are indeed mutually inverse in $\mathbf{F m}$. Hence $E A$ is isomorphic to $A$, and therefore $A$ is compact Hausdorff because $E A$ is. But when $A$ is compact Hausdorff, the inverse of $\eta_{A}$ is $\varepsilon_{A}$, so $\xi=\varepsilon_{A}$. This establishes that the $\mathbf{F m}^{\mathbb{E}}$-objects are precisely the pairs $\left(A, \varepsilon_{A}\right)$ with $A$ compact Hausdorff. Therefore $K$ is a bijection between the objects of $\mathbf{C H F m}$ and $\mathbf{F m}{ }^{\mathbb{E}}$ as claimed.

What we have been discussing is a manifestation of a general situation. If $\mathbf{C}$ is a reflective subcategory of $\mathbf{D}$ with associated monad $\mathbb{E}$, then the comparison functor $K: \mathbf{C} \rightarrow \mathbf{D}^{\mathbb{E}}$ is an isomorphism provided $\mathbf{C}$ is replete in $\mathbf{D}$, which means that it contains any $\mathbf{D}$-object that is isomorphic to a C-object. We just used the fact that $\mathbf{C H F m}$ is replete in $\mathbf{F m}$.

Without repleteness it can still be shown that $\mathbf{C}$ is equivalent to $\mathbf{D}^{\mathbb{E}}$. The equivalence is provided by the functors $K$ and $E \circ G^{\mathbb{E}}$. This follows because the above conclusion that $\eta_{A}$ and $\xi$ are mutually inverse can be lifted to show that $\eta_{A}$ is a morphism $(A, \xi) \rightarrow\left(E A, \mu_{A}\right)$ of $\mathbb{E}$-algebras with inverse $\xi:\left(E A, \mu_{A}\right) \rightarrow(A, \xi)$, and so $(A, \xi) \cong\left(E A, \mu_{A}\right)$ in $\mathbf{D}^{\mathbb{E}}$. The details are left to the interested reader.

\section{The Kleisli Category}

Let $\mathbf{C}(A, B)$ denote the "hom-set" of all arrows from object $A$ to object $B$ in category $\mathbf{C}$. The Kleisli category $\mathbf{F m}_{\mathbb{E}}$ has the same objects as $\mathbf{F m}$, with $\mathbf{F m}_{\mathbb{E}}(A, B)=\mathbf{F} \mathbf{m}(A, E B)$. In other words, an arrow from $A$ to $B$ in $\mathbf{F} \mathbf{m}_{\mathbb{E}}$ is given by an arrow from $A$ to $E B$ in $\mathbf{F m}$. To clarify this we will use the symbol $\leftrightarrow \rightarrow$ for an $\mathbf{F m}_{\mathbb{E}}$-arrow, and follow [8] by writing $f^{b}$ for the $\mathbf{F m}$-arrow $A \leftrightarrow B$ given by the Fm-arrow $f: A \rightarrow E B$. Particular care is needed in specifying the codomain of an $\mathbf{F m}_{\mathbb{E}}$-arrow because, for example, we know that $E B=E\left(B^{*}\right)$, so the same $f: A \rightarrow E\left(B^{*}\right)$ also determines an arrow $A \leftrightarrow B^{*}$ which will be different to the $A \leftrightarrow B$ one when $B \neq B^{*}$. This is 
analogous to the way that the identity function on the underlying set of a frame $A$ may generate two distinct arrows in $\mathbf{F m}$, the identity arrow $A \rightarrow A$ and the isomorphism $\sigma_{A}: A \rightarrow A^{*}$.

Given $f^{b}: A \leftrightarrow \rightarrow B$ and $g^{b}: B \leftrightarrow \rightarrow C$, composition in $\mathbf{F m}_{\mathbb{E}}$ is defined by $g^{b} \circ f^{b}=\left(\mu_{C} \circ E g \circ f\right)^{b}: A \odot C$, as in

$$
A \stackrel{f}{\longrightarrow} E B \stackrel{E g}{\longrightarrow} E E C \stackrel{\mu_{C}}{\longrightarrow} E C .
$$

The identity arrow on an $\mathbf{F m}_{\mathbb{E}}$-object $A$ is $\left(\eta_{A}\right)^{b}: A \leftrightarrow \rightarrow A$, given by $\eta_{A}$ : $A \rightarrow E A$.

There is a functor $G_{\mathbb{E}}: \mathbf{F m}_{\mathbb{E}} \rightarrow \mathbf{F m}$ having $G_{\mathbb{E}} A=E A$, and taking an arrow $f^{b}: A \leftrightarrow B$ to $\mu_{B} \circ E f:$

$$
E A \stackrel{E f}{\longrightarrow} E E B \stackrel{\mu_{B}}{\longrightarrow} E B .
$$

This has a left adjoint $F_{\mathbb{E}}: \mathbf{F m} \rightarrow \mathbf{F m}_{\mathbb{E}}$ with $F_{\mathbb{E}} A=A$, and taking $f: A \rightarrow$ $B$ to $\left(\eta_{B} \circ f\right)^{b}: A \odot B:$

$$
A \stackrel{f}{\longrightarrow} B \stackrel{\eta_{B}}{\longrightarrow} E B
$$

The adjoint pair $\left(F_{\mathbb{E}}, G_{\mathbb{E}}\right)$ also defines the monad $\mathbb{E}$ on $\mathbf{F m}$.

Now let $\mathbb{E}$ be the monad defined by our adjoint pair $(E, G)$ connecting Fm and CHFm. In the definition of $g^{b} \circ f^{b}$ we have $\mu_{C} \circ E g=\tilde{g}$ in the notation of Theorem 4 , since $\mu_{C}=\varepsilon_{E C}$. Thus

$$
g^{b} \circ f^{b}=(\tilde{g} \circ f)^{b} .
$$

A comparison functor $L: \mathbf{F m}_{\mathbb{E}} \rightarrow \mathbf{C H F m}$ is defined by $L A=E A$ on objects and $L\left(f^{b}\right)=\varepsilon_{E B} \circ E f$ on arrows $f^{b}: A \odot B$ :

$$
E A \stackrel{E f}{\longrightarrow} E E B \stackrel{\varepsilon_{E B}}{\longrightarrow} E B .
$$

This $L$ is the unique functor for which $G \circ L=G_{\mathbb{E}}$ and $L \circ F_{\mathbb{E}}=E$ :

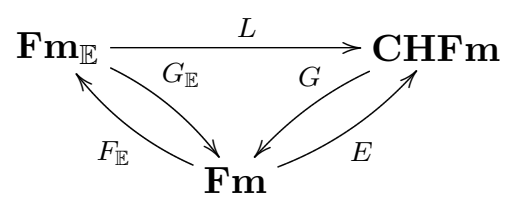

Again in the notation of Theorem $4, L\left(f^{b}\right)=\tilde{f}=$ the unique extension of $f: A \rightarrow E B$ along $\eta_{A}$. The existence and uniqueness of these arrows 
$\tilde{f}$ ensure that $L$ is full and faithful, which means that it acts bijectively in mapping any hom-set $\mathbf{F m}_{\mathbb{E}}(A, B)$ to $\mathbf{C H F m}(L A, L B)$. For if $L\left(f^{b}\right)=L\left(g^{b}\right)$, then $f=\tilde{f} \circ \eta_{A}=\tilde{g} \circ \eta_{A}=g$, and for any $h: L A \rightarrow L B$, if $f=h \circ \eta_{A}$, then we get $h=\tilde{f}=L\left(f^{b}\right)$.

The surjectivity of $L$ on hom-sets implies that the image of $L$ is the full subcategory of $\mathbf{C H F m}$ with collection of objects $\{E A: A$ is a frame $\}$. This is the category $\mathbf{U E F m}$ of all ultrafilter enlargements of frames, which was identified earlier as being equivalent to $\mathbf{C H F m}$ and to $\mathbf{D F m}$. $L$ is not an isomorphism of categories, since it is not injective on objects. For instance we know from Theorem 2(1) that $L A=L\left(A^{*}\right)$ for any frame $A$. But any full and faithful functor gives an equivalence between its domain and image categories [8, p. 91], so $\mathbf{F m}_{\mathbb{E}}$ and $\mathbf{U E F m}$ are equivalent. Since $\mathbf{C H F m}$ is isomorphic to the Eilenberg-Moore category $\mathbf{F m}^{\mathbb{E}}$, we see then that the five categories

\section{CHFm, DFm, UEFm, $\mathbf{F m}_{\mathbb{E}}, \mathbf{F m}^{\mathbb{E}}$}

are all equivalent, and are all dual to the category MA of modal algebras.

What has been described here for $\mathbf{C H F m}$ and $\mathbf{F m}$ is again quite general. Whenever $\mathbf{C}$ is a reflective subcategory of $\mathbf{D}$ with reflector $E$ determining monad $\mathbb{E}$, then the Kleisli category $\mathbf{D}_{\mathbb{E}}$ is equivalent to the Eilenberg-Moore category $\mathbf{D}^{\mathbb{E}}$. This is because $\mathbf{D}_{\mathbb{E}}$ is equivalent under $L$ to the image of $E$ in $\mathbf{C}$, which is itself equivalent $\mathbf{C}$ because each $\mathbf{C}$-object $A$ is isomorphic to $E A$, and $\mathbf{C}$ is in turn equivalent to $\mathbf{D}^{\mathbb{E}}$ under $K$. But for the frame case a stronger conclusion about $K$ was shown: $K$ is an isomorphism. We can also say something stronger about $L$ in this case: the image-closure operation $A \mapsto A^{*}$ can be used to show that $L$ is an equivalence.

The interest in this is that typically the construction of an equivalence out of a full and faithful functor may involve the axiom of choice (see [8, p. 94] or [2, p. 116]). Given an object $C$ in the image of $L$, choose some $A$ such that $C=L A$ and put $J C=A$. The bijective action of $L$ on hom-sets then inverts to allow $J$ to be defined on arrows to give a functor establishing an equivalence with $L$.

But there is no need to appeal to choice here, since we know from Theorem 2(2) that for any frames $A$ and $B$ we have $E A=E B$ only if $A^{*}=B^{*}$. Putting $J(E A)=A^{*}$ thus gives a well-defined function on UEFm-objects making $L(J(E A))=E\left(A^{*}\right)=E A . \quad J$ can be extended to arrows by noting that any UEFm-arrow $E A \rightarrow E B$ is equal to $\tilde{f}$ for a unique $f: A \rightarrow E B=E B^{*}$, so we can define $J(\tilde{f})=\left(f^{*}\right)^{b}: A^{*} \leftrightarrow \rightarrow B^{*}$. Since 
$E B$ is descriptive, Corollary 5 tells us that $\widetilde{f^{*}}=\tilde{f}$, so then

$$
L(J(\tilde{f}))=L\left(f^{* b}\right)=\widetilde{f^{*}}=\tilde{f},
$$

and hence $L \circ J=1_{\text {UEFm }}$.

From the other side, for each frame $A$ we have $J(L A)=A^{*}$. Now the functor $F_{\mathbb{E}}$ preserves isomorphisms (any functor does this), so the isomorphism $\sigma_{A}: A \rightarrow A^{*}$ in $\mathbf{F m}$ is sent by $F_{\mathbb{E}}$ to an isomorphism

$$
\left(\eta_{A^{*}} \circ \sigma_{A}\right)^{b}=\left(\eta_{A}\right)^{b}: A \odot A^{*}
$$

making $A \cong J(L A)$ in $\mathbf{F m}_{\mathbb{E}}$. (N.B: this is different to $\left(\eta_{A}\right)^{b}: A \leftrightarrow A$ when $A$ is not image-closed.)

On arrows, $J\left(L\left(f^{b}\right)\right)=J(\tilde{f})=\left(f^{*}\right)^{b}$. But the diagram

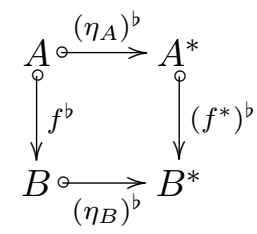

commutes in $\mathbf{F m}_{\mathbb{E}}$, because (using equation (III)):

$$
\left(f^{*}\right)^{b} \circ\left(\eta_{A}\right)^{b}=\left(\widetilde{f^{*}} \circ \eta_{A}\right)^{b}=\left(\tilde{f} \circ \eta_{A}\right)^{b}=f^{b},
$$

and

$$
\left(\eta_{B}\right)^{b} \circ f^{b}=\left(\widetilde{\eta_{B}} \circ f\right)^{b}=\left(1_{E B} \circ f\right)^{b} .
$$

Thus the isomorphisms $\left(\eta_{A}\right)^{b}$ are natural in $A$, showing that $J \circ L$ is naturally isomorphic to the identity functor on $\mathbf{F m}_{\mathbb{E}}$, and therefore that $J$ and $L$ form an equivalence between the categories $\mathbf{F} \mathbf{m}_{\mathbb{E}}$ and $\mathbf{U E F m}$.

\section{The Thomason Category}

Let CAMA be the full subcategory of MA whose objects are the complete and atomic modal algebras. It was shown in [9] that CAMA is dual to a category $\mathfrak{W}$ whose objects are the Kripke frames $\left(A, R^{A}\right)$, with a $\mathfrak{W}$-arrow $f$ from $\left(A, R^{A}\right)$ to $\left(B, R^{B}\right)$ being a function from the set $A$ to the set of all ultrafilters on the set $B$ such that

$$
\left\langle R^{B}\right\rangle X \in f(a) \quad \text { iff } \quad \exists b\left(a R^{A} b \text { and } X \in f(b)\right)
$$


for all $X \subseteq B$. Regarding a Kripke frame as a full frame in which every subset is internal, this condition equivalently states that

$$
f(a) \in\left\langle R^{E B}\right\rangle X^{E B} \quad \text { iff } \quad \exists b\left(a R^{A} b \text { and } f(b) \in X^{E A}\right) .
$$

But this asserts that $f$ is a modal map (Fm-arrow) from $A$ to $E B$. Thus $f$ can be identified with the Kleisli arrow $f^{b}: A \leftrightarrow \rightarrow B$. (Note that $E B$ itself will generally not be a full frame even when $B$ is.)

The identity $\mathfrak{W}$-arrow on full frame $A$ is given by $f(a)=\{X \subseteq A: a \in$ $X$ \}, which is $\eta_{A}(a)$, and so can be identified with the $\mathbf{F m}_{\mathbb{E}}$-identity $A \leftrightarrow A$. The composition of a $\mathfrak{W}$-arrow $f$ from $A$ to $B$ and a $\mathfrak{W}$-arrow $g$ from $B$ to $C$, i.e. two functions $f: A \rightarrow E B$ and $g: B \rightarrow E C$, is given by the function $g \bullet f: A \rightarrow E C$ having

$$
(g \bullet f)(a)=\{X \subseteq C:\{b \in B: X \in g(b)\} \in f(a)\} .
$$

Now $\left.\{b \in B: X \in g(b)\}=\left\{b \in B: g(b) \in X^{E C}\right)\right\}=g^{-1}\left(X^{E C}\right)$, so equation (II) tells us that $(g \bullet f)(a)=\tilde{g}(f(a))$. Therefore $g \bullet f=\tilde{g} \circ f$ and the Kleisli arrow $A \leftrightarrow C$ determined by $g \bullet f$ is $(\tilde{g} \circ f)^{b}$, which is $g^{b} \circ f^{b}$ by equation (III). Conclusion:

the Thomason category $\mathfrak{W}$ is the full subcategory of the Kleisli category $\mathbf{F m}_{\mathbb{E}}$ based on the Kripke frames.

Hence CAMA is dual to this subcategory of the Kleisli category.

\section{References}

[1] Michael Barr and Charles Wells. Toposes, Triples and Theories. Springer-Verlag, 1985.

[2] Francis Borceux. Handbook of Categorical Algebra 1. Basic Category Theory. Cambridge University Press, 1994.

[3] Francis Borceux. Handbook of Categorical Algebra 2. Categories and Structures. Cambridge University Press, 1994.

[4] Samuel Eilenberg and John C. Moore. Adjoint functors and triples. Illinois J. Math., 9:381-398, 1965.

[5] Robert Goldblatt. Metamathematics of Modal Logic. PhD thesis, Victoria University, Wellington, February 1974. Included in [6].

[6] Robert Goldblatt. Mathematics of Modality. CSLI Lecture Notes No. 43. CSLI Publications, Stanford, California, 1993. Distributed by Chicago University Press.

[7] H. Kleisli. Every standard construction is induced by a pair of adjoint functors. Proc. Amer. Math. Soc., 16:544-546, 1965. 
[8] Saunders Mac Lane. Categories for the Working Mathematician. Springer-Verlag, 1971.

[9] S. K. Thomason. Categories of frames for modal logic. The Journal of Symbolic Logic, 40:439-442, 1975.

\section{Robert GoldBlatt}

Centre for Logic, Language and Computation

Victoria University

P.O. Box 600, Wellington, New Zealand.

www.mcs. vuw.ac.nz/ ${ }^{\sim}$ rob

Rob.Goldblatt@mcs.vuw.ac.nz 